\title{
HuMAN B REgUlatory CELLS: THE NEW PLAYERS IN AUTOIMMUNE DISEASE
}

\author{
José L. Maravillas-Montero ${ }^{1 *}$ AND ERnesto AceVedo-Ochoa ${ }^{2}$ \\ ${ }^{1}$ Red de Apoyo a la Investigación, Universidad Nacional Autónoma de México and Instituto Nacional de Ciencias Médicas \\ y Nutrición Salvador Zubirán; ${ }^{2}$ Research Unit in Immunochemistry, Hospital de Especialidades del Centro Médico Nacional \\ "Siglo XXI", Instituto Mexicano del Seguro Social. Mexico City, Mexico
}

\begin{abstract}
Although the production of antigen-specific antibodies has been the originally accepted function of B-cells during immune responses, specific subsets that can negatively regulate inflammation, designated regulatory B-cells (Bregs), have been identified recently. These immunosuppressive cells support tolerance, mainly through the production of interleukin 10 and other unconventional factors. There have been emerging data suggesting their importance in diverse normal and pathologic processes. Novel and in development B-cell targeted therapies seem to be ideal treatments for different types of diseases such as cancer and allergy. Here, we discuss the current knowledge on the implication of Bregs in autoimmunity-related diseases, highlighting the importance of these cells for the development of novel strategies in the treatment of these pathologies.
\end{abstract}

Key words: B-cell. B regulatory cells. Autoimmunity.

\section{INTRODUCTION}

The immune system has evolved to deal with invading pathogens while at the same time sparing host tissues. It is not confined to a single organ or tissue but rather composed of specific cells and soluble compounds, such as the complement system, cytokines, chemokines, and antibodies.

Immune response-associated inflammation is essential for protecting the host against foreign pathogens; however, if left uncontrolled, it can cause deleterious and often irreversible tissue damage. Although initiation of any inflammatory response is mainly caused by injury or infection, a diversity of

Corresponding author:

*José L. Maravillas-Montero

Red de Apoyo a la Investigación

Instituto Nacional de Ciencias Médicas y Nutrición

Salvador Zubirán Vasco de Quiroga, 15

Col. Sección XVI, Del. Tlalpan

C.P. 14080, Ciudad de México, México

E-mail:maravillas@cic.unam.mx autoimmune diseases and cancer subtypes can promote chronic inflammation. Continuous exposure to pro-inflammatory stimuli contributes to the pathogenesis of numerous inflammatory and autoimmune disorders, including rheumatoid arthritis and lupus. To avoid irreversible damage, the prompt generation of an anti-inflammatory cellular response that minimizes injuries and promotes homeostasis is essential. Multiple inhibitory pathways that prevent cellular activation and restrain existing inflammatory signals promote these protective events. Accordingly, B lymphocytes represent a unique cell population that not only participates in pro-inflammatory reactions but also has roles in the regulation of immune-related processes.

Received for publication: 25-04-2017

Accepted for publication: 29-05-2017

doi: $10.24875 /$ RIC.17002266 
B lymphocytes, or B-cells, represent the arm of the adaptive immune response termed humoral immunity. They are mainly derived from common lymphoid progenitors in the bone marrow, where they develop from pro-B cells to pre-B cells and finally to immature B-cells before they are let out into the periphery as transitional cells. When these immature transitional B-cells reach the spleen, they go through more advanced transitional stages before taking on their final phenotype as fully mature B-cells. After activation, mature B-cells can produce cytokines and present antigen to T-cells, but their hallmark feature and main function, once they are terminally differentiated into plasma cells, is the production of soluble antibodies ${ }^{1}$.

Mature B-cells can be subdivided into subsets that are distinguishable in terms of their development, phenotype, function, and anatomical localization. Among these, several regulatory B-cell subsets (collectively designated as Bregs) are now being recognized and have been recently characterized, particularly those present in humans (Table 1 ), although their origins and relations to each other are not completely clear $^{1-3}$.

Bregs are important in the maintenance of tolerance and immune homeostasis although they represent < $10 \%$ of circulating B-cells in healthy individuals. Bregsmediated suppression occurs primarily through the production of interleukin $10(\mathrm{IL}-10)^{4}$. In addition to this cytokine, several other Bregs immunosuppressive mechanisms have been identified (Fig. 1). Thus, the gold standard for their identification is the assessment of their suppressive function by limiting effector mechanisms in target cells exerted through cytokines as well as direct contact with them (Fig. 1 and Table 1).

New approaches toward understanding Bregs biology in health and disease will provide new opportunities to develop Bregs immunotherapy that could prove beneficial for the treatment of various pathologies associated to an immune component.

Functional and numerical alterations in Bregs have been reported in several autoimmune diseases where inverse correlations between Bregs activity and disease severity are present ${ }^{3}$.

A suppressive B-cell subset was first identified in helminth-infected patients with multiple sclerosis (MS) who displayed an increased frequency of $\mathrm{CD} 19^{+} \mathrm{CD} 1 \mathrm{~d}^{\mathrm{hi}}$ IL-10-producing B-cells along with a better clinical outcome. In addition, in relapsing-remitting MS (RRMS), a decrease in IL-10-producing B-cells was detected in patients undergoing relapse compared with patients in remission and with healthy individuals. Remarkably, treatments for RRMS consisting of interferon (IFN- $\beta$ ), fingolimod (a sphingosine 1-phosphate modulator), or alemtuzumab (anti-CD52) have demonstrated positive effects through the expansion of CD2 $44^{\text {hi }} C D 38^{\text {hi }}$ $\mathrm{B}$ regulatory population. In this way, it becomes clear that immunomodulatory treatments in MS could function through the induction of a shift in specific B-cell subsets toward a more anti-inflammatory phenotype ${ }^{5}$.

In systemic lupus erythematosus (SLE) patients, the inability of immature $\mathrm{CD} 19^{+} \mathrm{CD} 24^{\mathrm{hi}} \mathrm{CD} 38^{\mathrm{hi}}$ B-cells to differentiate into Bregs has been detected together with an impaired IL-10-producing ability upon CD40 activation, affecting in this way, any Th1 response-suppression effect ${ }^{2,3}$.

SLE is characterized by an IFN- $\alpha$-induced signature that impacts in immature B-cell differentiation. It has been shown that low concentrations of IFN- $\alpha$ simultaneously expands populations of both plasmablasts and Bregs; conversely, high levels of this cytokine promote B-cell

Table 1. Human B (CD19+) regulatory cell subsets

\begin{tabular}{|c|c|c|c|}
\hline Subset name & Phenotype & Regulatory molecules & Target cells \\
\hline Immature B-cells & CD2 $4^{\text {hi }}$ CD $38^{\text {hi }}$ & IL-10, PD-L1, CD80, CD86 & $\mathrm{CD}^{+}{ }^{+} \mathrm{T}$-cells, CD8 ${ }^{+} \mathrm{T}$-cells, pDCs, iNKT cells \\
\hline B10 cells & $\mathrm{CD} 24^{\mathrm{hi}}, \mathrm{CD} 27^{+}$ & $\mathrm{IL}-10$ & Monocytes \\
\hline iBregs & Undefined & TGF- $\beta$, IDO & CD4 ${ }^{+} \mathrm{T}$-cells \\
\hline GzmB + B-cells & $\mathrm{CD}^{3} 8^{+}, \mathrm{CD} 1 \mathrm{~d}^{+}, \mathrm{IgM}^{+}, \mathrm{CD} 147^{+}$ & GzmB+, IL-10, IDO & CD4 ${ }^{+} \mathrm{T}$-cells \\
\hline Br1 cells & CD25 hi, CD71 $1^{\text {hi }}$, CD73 $3^{\text {lo }}$ & IL-10, IgG4 & CD4 ${ }^{+} \mathrm{T}$-cells \\
\hline NA & $\mathrm{CD}^{\circ} 9^{+}, \mathrm{CD}^{+} 3^{+}$ & Adenosine & CD4 ${ }^{+} \mathrm{T}$-cells, CD8 ${ }^{+} \mathrm{T}$-cells \\
\hline NA & $\mathrm{TIM}_{1}^{+}$ & $\mathrm{IL}-10$ & $\mathrm{CD}^{+}{ }^{+} \mathrm{T}$-cells, $\mathrm{CD} 8^{+} \mathrm{T}$-cells \\
\hline
\end{tabular}

PD-L1: programmed death-ligand 1; pDCs: plasmacytoid dendritic cells; iNKT: invariant natural killer T-cells; TGF- $\beta$ : transforming growth factor $\beta$; IDO: Indoleamine-pyrrole 2,3-dioxygenase; GzmB: granzyme B; TIM1: T-cell immunoglobulin and mucin domain-containing protein-1; IgG4: immunoglobulin 4; NA: not available 
Figure 1. Human B regulatory cells (Bregs) effector mechanisms. Bregs can secrete different anti-inflammatory cytokines such as interleukin 10 (IL-10), transforming growth factor $\beta$ and IL-35. They also express surface molecules includig CD80, CD86, or PD-L1 that mediates suppression by direct cell-cell interactions. Bregs can also use enzymes to exert its effects in target cells, these are for example intracellular granzyme B or indoleamine-pyrrole 2,3-dioxygenase or the membrane-expressed CD73 that mediates the generation of adenosine. Finally, some Bregs subsets can also switch to produce immunoglobulin 4 after activation; a secreted immunoglobulin also related with immune-suppressive functions. Collectively, these elements can suppress the functionality of lymphoid (inhibiting for example the IL-17 or interferon- $\gamma$ production by Th17, Th1 or T CD8 ${ }^{+}$cells) or myeloid (preventing the secretion of tumor necrosis factor- $\alpha$ or IL-12 by inflammatory monocytes or dendritic cells, respectively). In addition, Bregs can also enhance the effector abilities of Bregs (promoting IL-10 secretion) and invariant natural killer cells (inducing them to produce IL-4 and IL-13) to favor an anti-inflammatory microenvironment.

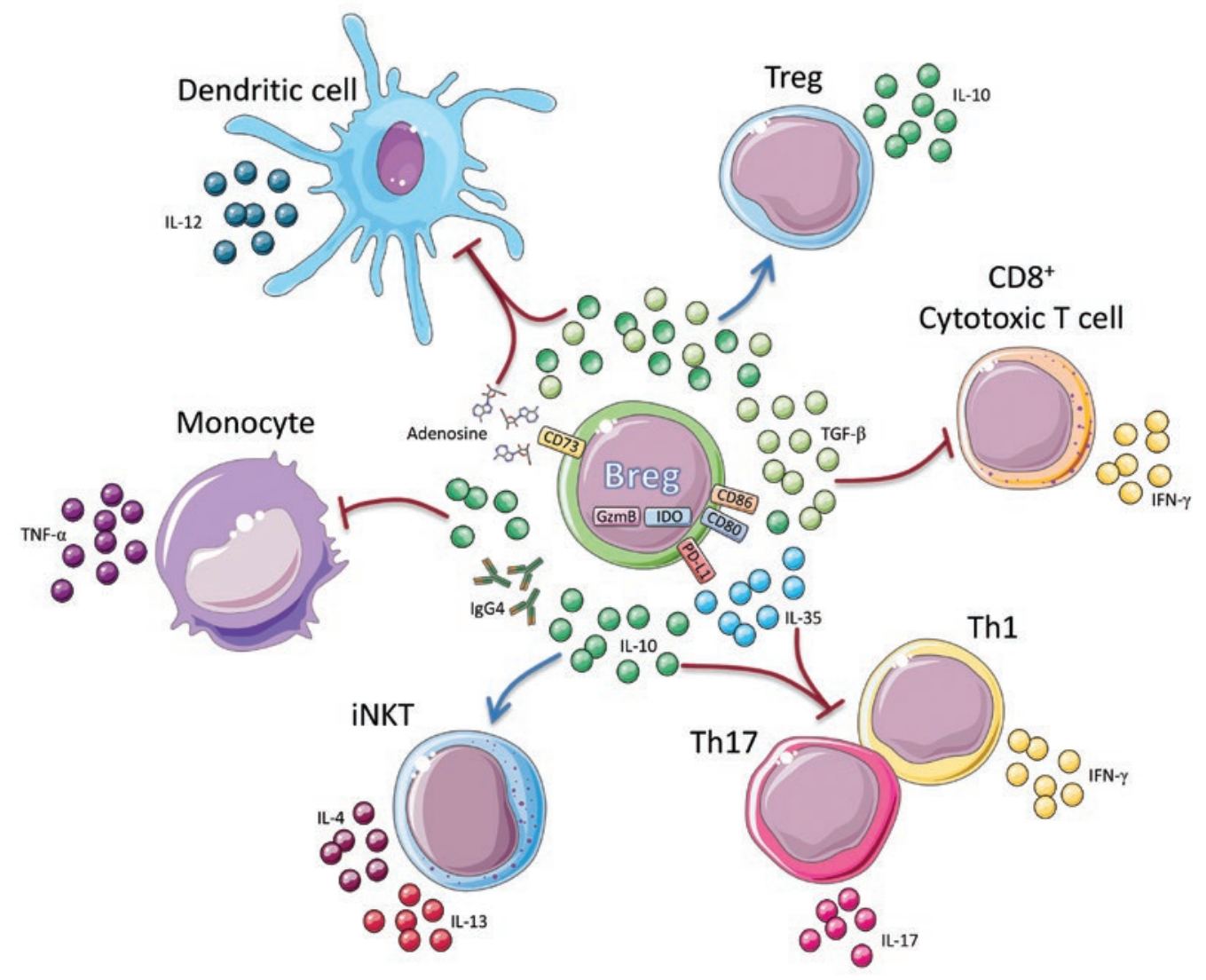

differentiation in favor of plasmablasts but fail to support the expansion of Bregs. Furthermore, functionally weakened CD24 $4^{\text {hiCD}} 38^{\text {hi }}$ Bregs isolated from SLE patients fail to prevent IFN- $\alpha$ production by plasmacytoid dendritic cells, one of the main sources of Type IIFNs ${ }^{6}$.

Interestingly, when SLE patients are treated with rituximab (inducing B-cell depletion) and after B-cell number's recovery, a higher immature-to-memory cell population's ratio has been associated with long-term remission, suggesting that repopulation with $\mathrm{CD} 24^{\text {hi }} \mathrm{CD} 38^{\text {hi }}$ Bregs could be related with an improved clinical response ${ }^{3,4}$.

Numerous studies reported reduced frequencies of B10 cells, CD24 ${ }^{\text {hi } C D} 38^{\text {hi }}$ Bregs, IL-10+TIM1+ $\mathrm{B}$-cells, and $\mathrm{IL}-10^{+} \mathrm{CD} 5^{+} \mathrm{CD} 1 \mathrm{~d}^{\text {hi }} \mathrm{B}$-cells in rheumatoid arthritis patients compared with healthy controls. Similar reports arise involving patients with Type 1 diabetes, psoriasis, and inflammatory bowel disease, where a decrease in suppressive IL-10-producing Bregs numbers has been linked to disease progression?

As showed previously, current knowledge is promoting the establishment of Bregs as key regulators of autoimmune diseases, but their importance is not limited to these entities. $C D 24^{\text {hi }} \mathrm{CD} 38^{\text {hi }}$ Bregs seem to play an important role in the development of transplant tolerance by suppressing effector T-cell responses. Studies involving allogeneic hematopoietic stem-cell transplant recipients showed that Bregs from patients with chronic graft-versus-host disease (cGVHD) exhibited reduced IL-10 secretion compared with non-cGVHD 
patients and healthy individuals. Correspondingly, increased $C D 24^{\text {hi }} C D 38^{\text {hi }}$ B-cell numbers were found to be associated with reduced renal transplant rejection rates ${ }^{8}$.

Most of the treatments for autoimmune pathologies control the symptoms of the diseases rather than offer definitive cures. In addition, these treatments usually become toxic and unsuccessful when they are administered over a long period. In addition, long-term use of immunosuppressive drugs and steroids increases the risk of severe infections. Considering these observations, cellular immunotherapy acquires attractiveness as it could offer a highly-targeted deletion or modulation of pathology-generating cells. Since Bregs represent important modulators of the immune response and promote immunological tolerance, strategies focused on isolating, in vivo or ex vivo expanding and infusing these B-cell subsets would constitute a new approach to control or even possibly cure numerous immune-mediated illnesses.

Current evidence indicates that the microenvironment is a crucial factor for induction of Bregs. Besides toll-like receptors (TLRs), CD40, and B-cell receptor-induced signaling, which are imperative for Bregs functions, there is emerging evidence suggesting that inflammatory cytokines perform a critical role in the immunosuppressive abilities of these B lymphocytes. Thus, an expansion of Bregs has been detected upon stimulation by IFN- $\alpha$, IFN- $\beta$, IL-1 $\beta, \mathrm{IL}-6$, and B-cell activating factor, where TLRs or CD40 stimulation synergizes to enhance this phenomenon. Although these data support the possibility of expansion of Bregs in vivo, the systemic administration of inflammatory cytokines could generate undesirable pro-inflammatory responses from several cell types, deriving into serious adverse effects that must be taken into consideration. Besides that, the possibility to differentiate effector inflammatory B-cells instead of Bregs using high doses of these cytokines is always present. Furthermore, it has been observed that in autoimmune disease patients, chronic exposure of B-cells to elevated levels of inflammatory cytokines results in a reduction of the functional Bregs population that is incompetent for tolerance restoration ${ }^{3,4,9,10}$.

Surprisingly, and according to recent findings related to other immune system components, microbiota has also been reported to play a role in Bregs' generation, at least in rodent models. Decreased numbers of Bregs were found in antibiotic-treated mice when compared with untreated individuals. Furthermore, in arthritic mice, gut commensal bacteria-induced IL-1 $\beta$ and IL-6 secretion-enhanced Bregs differentiation in conjunction with IL-10 production. Thus, better understanding of the signals that drive Bregs' generation could deliver new and improved approaches for the in vivo potential of Bregs therapies ${ }^{3}$.

\section{CONCLUSION}

Clearly, Bregs are critical for tolerance maintenance and for the suppression of inflammation. Bregstargeted therapies, aside from their potential risks or the lack of understanding of their mechanisms of action, now emerge as a challenging treatment option that shows great promise and could represent a new tactic to deal with immune-related diseases.

\section{ACKNOWLEDGMENTS}

This work was supported by grants 240314 from CONACYT and IA204316 from UNAM-DGAPA-PAPIIT Program. The authors want to thank to David E. Meza Sánchez for reviewing the manuscript. E.A.O. received the fellowship number 607542 from CONACYT and 99097182 from IMSS.

\section{REFERENCES}

1. Hoffman W, Lakkis FG, Chalasani G. B cells, antibodies, and more. Clin J Am Soc Nephrol. 2016;11:137-54.

2. Gorosito Serrán M, Fiocca Vernengo $F$, Beccaria CG, Acosta Rodriguez EV, Montes CL, Gruppi A. The regulatory role of $B$ cells in autoimmunity, infections and cancer: Perspectives beyond IL10 production. FEBS Lett. 2015;589:3362-9.

3. Miyagaki T, Fujimoto M, Sato S. Regulatory B cells in human inflammatory and autoimmune diseases: From mouse models to clinical research. Int Immunol. 2015;27:495-504

4. Ray A, Dittel BN. Mechanisms of regulatory B cell function in autoimmune and inflammatory diseases beyond IL-10. J Clin Med. 2017;6. pii: E12.

5. Han J, Sun L, Fan X, Wang Z, Cheng Y, Zhu J, et al. Role of regulatory b cells in neuroimmunologic disorders. J Neurosci Res. 2016;94:693-701.

6. Menon M, Blair PA, Isenberg DA, Mauri C. A regulatory feedback between plasmacytoid dendritic cells and regulatory $B$ cells is aberrant in systemic lupus erythematosus. Immunity. 2016;44:683-97

7. Bugatti S, Vitolo B, Caporali R, Montecucco C, Manzo A. B cells in rheumatoid arthritis: From pathogenic players to disease biomarkers. Biomed Res Int. 2014;2014:681678

8. Sarvaria A, Basar R, Mehta RS, Shaim H, Muftuoglu M, Khoder A, et al. IL-10 regulatory $B$ cells are enriched in cord blood and may protect against cGVHD after cord blood transplantation. Blood. 2016;128:1346-1.

9. Ray A, Wang L, Dittel BN. IL-10-independent regulatory B-cell subsets and mechanisms of action. Int Immunol. 2015;27:531-6.

10. van de Veen W, Stanic B, Wirz OF, Jansen K, Globinska A Akdis $M$. Role of regulatory $B$ cells in immune tolerance to allergens and beyond. J Allergy Clin Immunol. 2016;138:654-5. 